\title{
SPECTROSCOPIC CCD STUDY OF THE TWO-DAY WN5+O8V BINARY CX CEP
}

\author{
DAVID LEWIS, ANTHONY F. J. MOFFAT, and CARMELLE ROBERT \\ Département de physique, Université de Montréal, Montréal and Observatoire du mont \\ Mégantic, Canada
}

After CQ Cep, the system CX Cep has the shortest orbital period known among Galactic $\mathrm{WR}+\mathrm{O}$ binaries. However, no definitive spectroscopic study is yet available for CX Cep, probably because of its relatively faint magnitude $(B \approx 13)$. We have therefore obtained and analyzed some 60 CCD spectra (3700-4900 $\AA, \mathrm{S} / \mathrm{N} \simeq 100,5 \AA / 2$ pixels) in August and October 1987.

Phased radial velocities (RV) show circular orbits with emission and absorption lines generally moving in antiphase. The best estimate of the RV amplitudes are $K_{\mathrm{WR}}=340 \pm 10 \mathrm{~km} \mathrm{~s}^{-1}$ (mainly from NV 4603) and $K_{\mathrm{O}}=240 \pm 15 \mathrm{~km} \mathrm{~s}^{-1}$ (from Balmer and Pickering absorption lines). With orbital inclination $74^{\circ} \pm 5^{\circ}$ from polarization observations (Shulte-Ladbeck and van der Hucht 1989, Ap. J., 337, 872) we find the masses $M_{\mathrm{WR}}=20 \pm 5 M_{\odot}$ and $M_{\odot}=28 \pm 7 M_{\odot}$ and the orbital separation $a=25 \pm 2 R_{\odot}$. With core radius $R_{\mathrm{WN} 5} \sim 3 R_{\odot}$ and $R_{\mathrm{O} 8 \mathrm{~V}} \sim 9 R_{\odot}$, the system is therefore not in contact.

Nevertheless, the relatively close orbit does produce interaction effects as illustrated in the Figure. Phased equivalent widths of emission lines show that:

(a) NIV 4058 is reduced in strength at phase 0.5 (O star in front) probably by a simple eclipse effect of the NIV emitting part of the WR wind;

(b) NV 4603/19 is weaker at both phases 0.5 and 0.0 implying that much of the NV emission must arise between the two stars (via wind collision?);

(c) HeII 4686 (emission) is strongest near phase 0.4, when the $O$ star is still approaching the observer (bow shock effect?).
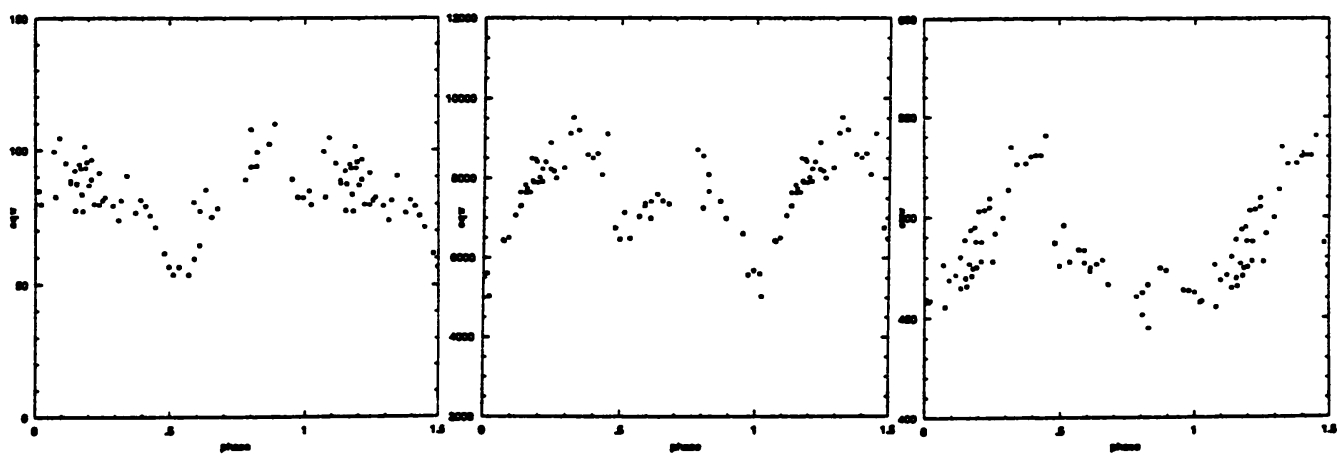

FIGURE. Equivalent width versus phase (WR behind at $\varphi=0.5$ ) of emission lines NIV 4058, NV 4603/19 and HeIl 4686 (left to right).

K. A. van der Hucht and B. Hidayat (eds.),

Wolf-Rayet Stars and Interrelations with Other Massive Stars in Galaxies, 256.

(C) 1991 IAU. Printed in the Netherlands. 